\title{
Boundary-integral model of permanent magnet of a tube segment as shape
}

\author{
Krystyn PaWluk, Renata Sulima \\ Electrotechnical Institute \\ Department of Electric Machines \\ Warszawa \\ e-mail:pawluk@iel.waw.pl
}

(Received: 23.05.2011, revised 01.08.2011)

\begin{abstract}
The magnetic field due to a permanent magnet of a tube-side segment as shape and of radial-oriented magnetization is considered. Such a sheet modelling a single pole of the magnet is used to express the suitable contribution to magnetic quantities. A boundary-integral approach is applied that is based on a virtual scalar quantity attributed to the magnet pole. Such an approach leads to express analytically the scalar magnetic potential and the magnetic flux density by means of the elliptic integrals. Numerical examples of the computed fields are given. The general idea of the presented approach is mainly directed towards designing the magnetic field within the air gap of electric machines with permanent magnets as an excitation source. Other technical structures with permanent magnets may be a subject of this approach as well.
\end{abstract}

Key words: permanent magnets, boundary-integral method, elliptic integrals

\section{Introduction}

Rotating electric machines with permanent magnets of both motor and generator type have a growing use, nowadays. Modern permanent high-energy magnets with almost linear $B(H)$ curve can produce the magnetic field exciting effectively such a machine. Contrary to an electromagnetic excitation, on which the classical design calculation of electric machines is based, an appropriate attempt for the corresponding design in the case of the permanentmagnet excitation seems to be of special interest, mainly when the magnetic field distribution through a machine air gap is under consideration.

A main goal of the present paper is to study the magnetic field exerted by the permanent magnet of typical shape representing a segment of a tube-wall with radial magnetization that is applied for synchronous machines of radial air-gap flux, see Figure 1. The boundary-integral model of the permanent magnet can be proposed for design computation of permanent-magnet machines. Such a model may be regarded as a simpler alternative to the finite elements method (that is employed and implemented in various professional software), the use of which 
for specialized design program of electric machines causes some difficulties in application of the 3-D net to the machine geometrical structure and also in interpretation of the magnetic field within the air gap being virtually subdivided into numerous volume elements of irregular shape.

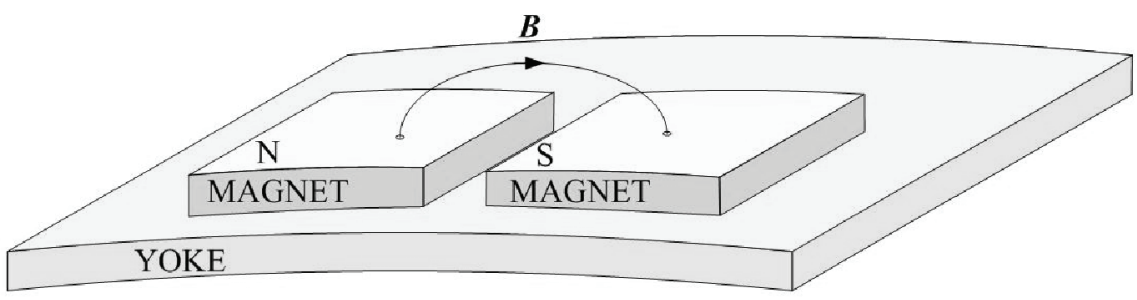

Fig. 1. Permanent magnet of a tube segment as shape stuck to a yoke

The model we present here is deprived of such drawbacks, hence the 3-D net is not necessary in the boundary-integral approach. The issue of the boundary-integral model of permanent magnet [3-6, 12] is focussed on a concept of the virtual boundary quantity $\sigma_{\mathrm{m}}$ that we define by a "discontinuity jump" expressed by a difference between two components of the magnetic polarization $J_{n}=\boldsymbol{e} \cdot \boldsymbol{J}$ that are normal to the magnet pole surface on both sides of it, i.e.

$$
\sigma_{\mathrm{m}}=J_{(-)}-J_{(+)},
$$

where $J_{(-)}$and $J_{(+)}$are the above components of magnetic polarization on the pole surface. We have the following boundary densities for the $\mathrm{N}$ - and S-pole of the homogeneously magnetized permanent magnet being put in free space:

$$
\begin{gathered}
\sigma_{\mathrm{mN}}=J_{\mathrm{N}(-)}-J_{\mathrm{N}(+)}=J-0=J, \\
\sigma_{\mathrm{mS}}=J_{\mathrm{S}(-)}-J_{\mathrm{S}(+)}=0-J=-J .
\end{gathered}
$$

If the S-pole surface of the magnet sticks firmly to a ferromagnetic yoke, equation (2) determines $\sigma_{\mathrm{mN}}$ on the external side of the magnet and on its yoke side the following equation holds $\sigma_{\mathrm{mS}}=J_{\text {yoke }}-J \approx B_{\text {yoke }}-J$. For approximate evaluating the magnetic field in the air gap of an electric machine a super permeable yoke may be assumed to which $\sigma_{\mathrm{ms}} \approx 0$ corresponds.

Let us attribute the term surface density of magnetic charge ${ }^{1}$ to the quantity $\sigma_{\mathrm{m}}$ that is a fictitious single layer magnetic density involving the scalar magnetic potential. A usable out-

\footnotetext{
${ }^{1}$ The term surface density of magnetic charge awakes a room of doubt, as a concept of magnetic charge is not accepted in physics. However, this quantity of surface-density character engenders the magnetic field conform to the same principle as the surface density of electric charge generates the electric field. Craik [2] avoids this terminological problem using an enigmatic term pole strength for the quantity $\sigma=M_{n}$ where $M_{n}$ is an outward component of magnetization. Our concept of quantity $\sigma_{\mathrm{m}}$ corresponds to $\mu_{0} \sigma$ of Craig. It is worth to keep in mind that the common surface integral of $\sigma_{\mathrm{m}}$ over both magnet pole surfaces, where the first $\sigma_{\mathrm{m}}$ is constant positive and the second one constant negative, gives zero. Thus,
} 
line of the boundary-integral approach is termed boundary element method. In a corresponding software, the numerical integrations are performed over the 2-D boundary elements that subdivide the boundaries of an examined object. We have shown in [7-9] that for the box-shape permanent magnets the analytical integration over the both magnet-pole rectangular surfaces can be applied and, moreover, it leads to correct results even in close distance from the magnet poles. A similar advantage can be reached for the magnet shape formed as a segment of a tube with radial magnetization. A mathematical procedure leading to obtaining a distribution in space, the magnetic flux density consists in calculation of the integrals over two (N and $\mathrm{S}$ ) thin cylindrical magnet-pole sheets on which $\sigma_{\mathrm{m}}$ is homogeneously distributed, hence, this approach seems to be also suitable for computing the magnetic field within the air gap of electric machines or of other apparatuses equipped with magnets of such a shape.

\section{Boundary-integral approach}

Two kinds of field sources are considered in the boundary-integral approach: primary and secondary sources, see [3, 12]. Regarding an electric machine, the primary are of double type:

- $\sigma_{\mathrm{m}}-$ surface densities upon the both magnet-pole surfaces,

- $\Theta$ - current linkage of armature winding of an electric machine supplied with an electric current depending on output of the machine.

At no-load, only the first of them is taken into account. The secondary sources are attributed to the boundary between the air and ferromagnetic core of the armature; it is approximated by:

$$
\sigma_{\mathrm{m} 2}=\left(1 / \mu_{\mathrm{r}}-1\right) B_{n} \approx B_{n},
$$

where $\mu_{r}$ is relative permeability of the ferromagnetic material and $B_{n}$ is magnetic flux density normal to the boundary that would be engendered by the primary sources at the positions of the boundary under fictitious assumption that the ferromagnetic part is removed from the magnetic structure. For the air gap of electric machine such a boundary is formed by heads of armature teeth and bottoms of slots. Following the above, the boundary-integral algorithm for computing the magnetic field within an air gap of electric machine should consist of 2 steps given bellow, the idea of which is schematically marked in Figure 2 representing a permanent magnet and a current linkage localized within a machine slots (the cylindrical shape of the corresponding elements is in the figure neglected).

1) To compute preliminary $B_{n}$ (i.e. the radial component $B_{r}$ of $\vec{B}$ in the machine co-ordinate system) that depends not otherwise than on the primary sources $\left(\sigma_{\mathrm{mN}}, \sigma_{\mathrm{mS}}, \Theta\right.$ in Figure 2a). It should be done only at field points situated on the positions of the heads of armature teeth and of bottoms of slots. It is supposed that the armature core is taken away (that is symbolized by the dashed line in Figure 2a), and, following (4), to interpret the $B_{n}, B_{n}^{\prime}$,

the total "magnetic charge" of the permanent magnet becomes really zero and we can conclude that using correctly a concept of magnetic charge density as a virtual pole-sheet quantity we are not in conflict with the physical understanding of the magnetic phenomena, indeed. 
$B_{n}^{\prime \prime}$, as the secondary source $\sigma_{\mathrm{m} 2}$ (in particular $\sigma_{\mathrm{m} 2}, \sigma_{\mathrm{m} 2}^{\prime}, \sigma_{\mathrm{m} 2}^{\prime \prime}$ in Figure $2 \mathrm{~b}$ ), being of opposite sign then $\sigma_{\mathrm{mN}}$.

a)

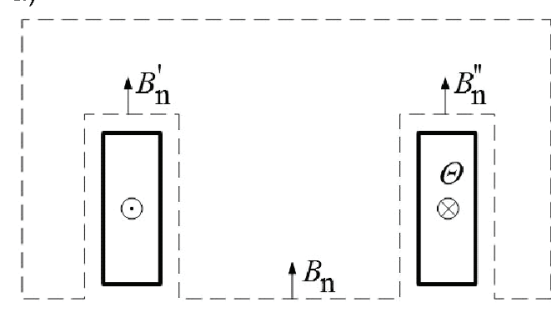

$\mathrm{N}$

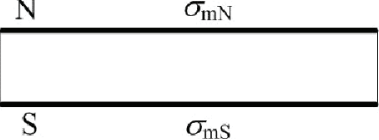

b)

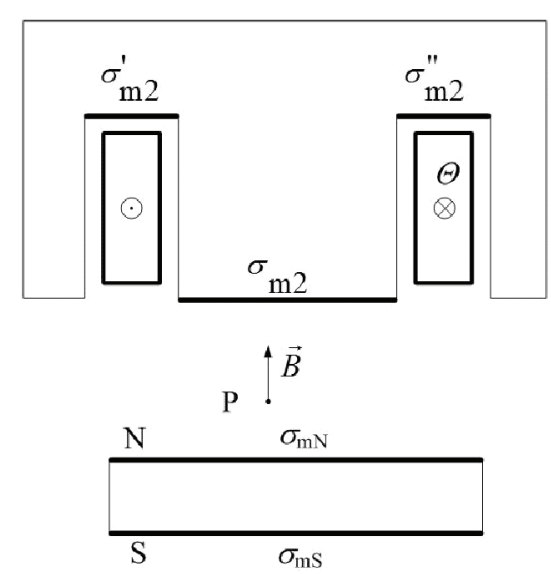

Fig. 2. Idea of computing the components of $\boldsymbol{B}$ within the air gap of an electric machine with permanent magnets, by means of the primary and secondary sources of $\sigma_{\mathrm{m}}$

2) To compute the finite values of $\boldsymbol{B}$ at the field points $P$ of consideration within the air gap and being expressed by means of its particular vector components $e_{r} B_{r}, e_{\theta} B_{\theta}, e_{z} B_{z}$, where the first one is of primordial interest. The result values of components of $\boldsymbol{B}$ are superposed by both the primary sources (i.e. by $\sigma_{\mathrm{mN}}, \sigma_{\mathrm{ms}}, \Theta$ ) and by the secondary ones $\left(\sigma_{\mathrm{m} 2}, \sigma_{\mathrm{m} 2}^{\prime}, \sigma_{\mathrm{m} 2}^{\prime \prime}\right)$ that increase the primary-sources field.

The subscripts $r, \theta, z$ determine the radial, azimuthal and axial components of $\boldsymbol{B}$, respectively. The formulae of the particular components exerted by $\sigma_{\mathrm{m}}$ are the main subject of the paper. A basic geometric pattern, on which the idea of computing the magnetic field under consideration is developed, is a single cylindrical sheet with $\sigma_{\mathrm{m}}=$ const.

The model sketch of the sheet is shown in Figure 3. The cylindrical co-ordinate system with origin at 0 is applied. The sheet is characterized by radius $\rho$, extension arc $2 \vartheta_{0}$ and length $2 l_{0}$. The model is similarly valid for both permanent magnet pole surfaces as well as for the interface between a ferromagnetic core of the machine and its air gap. For the latter case, $2 \vartheta_{0}$ represents the extension arc of a tooth head and a slot bottom; the surface density $\sigma_{\mathrm{m} 2}$ is attributed on these boundaries. The points $P(r, \theta, z)$ and $\mathrm{Q}\left(r^{\prime}, \theta^{\prime}, z^{\prime}\right) \equiv \mathrm{Q}(\rho, \vartheta, \zeta)$ represent the field and source points, respectively. The source points $\mathrm{Q}$ are localized at the sheet; the field points $\mathrm{P}$ are everywhere in space. The model is valid for the electric machine with both the inner or outer field system. In the first case the point $\mathrm{P}$ is located at the convex side of the sheet $(r>\rho)$ and for the second case at its concave side $(r<\rho)$.

Let us note that to determine the scalar magnetic potential at P due to $\sigma_{\mathrm{m}}$ at $\mathrm{Q}$, the Green function of the sheet model is $\mathrm{G}=f\left(\left|\boldsymbol{R}_{\mathrm{P}}-\boldsymbol{R}_{\mathrm{Q}}\right|\right)$, where $\boldsymbol{R}_{\mathrm{P}}, \boldsymbol{R}_{\mathrm{Q}}$ are position vectors of points 


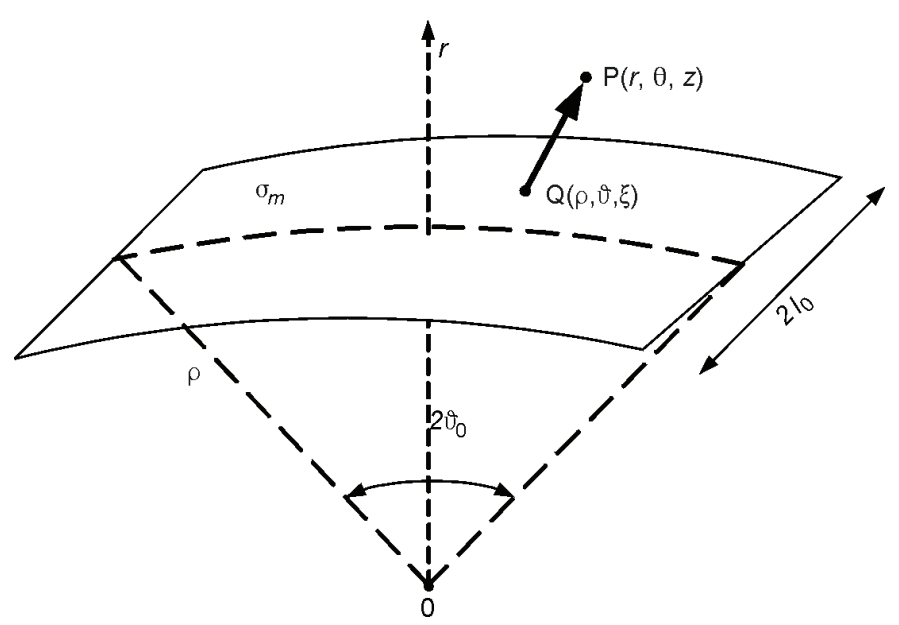

Fig. 3. Model of a cylindrical sheet with $\sigma_{\mathrm{m}}$ to compute the magnetic field at $P$

$P$ and $Q$, respectively. The norm of this vector difference represents an Euclidean distance between $P$ and $Q$ that in the cylindrical co-ordinate system is expressed by:

$$
\left|\boldsymbol{R}_{P Q}\right|=\sqrt{r^{2}+\rho^{2}-2 r \rho \cos (\theta-\vartheta)+(z-\zeta)^{2}}
$$

hence, the scalar magnetic potential at $\mathrm{P}$ can be defined, as a quantity proportional to the inverse of $\left|\boldsymbol{R}_{P Q}\right|$, by the following double integral over the sheet surface, i.e. it can be given by:

$$
\psi_{m}(r, \theta, z)=C_{\psi} \int_{-l_{0}}^{l_{0}} \int_{-\vartheta_{0}}^{\vartheta_{0}} \frac{\rho \mathrm{d} \vartheta \mathrm{d} \zeta}{\left|\boldsymbol{R}_{P Q}\right|}=C_{\psi} \int_{-l_{0}}^{l_{0}} \int_{-\vartheta_{0}}^{\vartheta_{0}} \frac{\rho \mathrm{d} \vartheta \mathrm{d} \zeta}{\sqrt{r^{2}+\rho^{2}-2 r \rho \cos (\theta-\vartheta)+(z-\zeta)^{2}}},
$$

where $C_{\psi}=\sigma_{m} /\left(4 \pi \mu_{0}\right)$. The above surface integral is non-singular everywhere except the point $\mathrm{Q}(\zeta \rightarrow z ; \vartheta \rightarrow \theta)$ for $R=\rho$.

The formulas of the cylindrical components of magnetic flux density $\boldsymbol{B}$ can be reached by the relevant differentiations of the magnetic scalar potential (6) with respect to the following co-ordinates of $P$ to read:

$$
\begin{aligned}
& B_{r}(r, \theta, z)=-\mu_{0} \frac{\partial \psi_{\mathrm{m}}}{\partial r}=C_{B} \int_{-l_{0}}^{l_{0}} \int_{-\vartheta_{0}}^{\vartheta_{0}} \frac{[r-\rho \cos (\theta-\partial)] \rho \mathrm{d} \vartheta \mathrm{d} \zeta}{\sqrt{\left[r^{2}+\rho^{2}-2 r \rho \cos (\theta-\vartheta)+(z-\zeta)^{2}\right]^{3 / 2}}}, \\
& B_{\theta}(r, \theta, z)=-\mu_{0} \frac{\partial \psi_{\mathrm{m}}}{r \partial \theta}=C_{B} \int_{-l_{0}}^{l_{0}} \int_{-\vartheta_{0}}^{\vartheta_{0}} \frac{\sin (\theta-\partial) \rho^{2} \mathrm{~d} \vartheta \mathrm{d} \zeta}{\sqrt{\left[r^{2}+\rho^{2}-2 r \rho \cos (\theta-\vartheta)+(z-\zeta)^{2}\right]^{3 / 2}}}, \\
& B_{z}(r, \theta, z)=-\mu_{0} \frac{\partial \psi_{\mathrm{m}}}{r \partial \theta}=C_{B} \int_{-l_{0}}^{l_{0}} \int_{-\vartheta_{0}}^{\vartheta_{0}} \frac{(z-\xi) \rho \mathrm{d} \vartheta \mathrm{d} \zeta}{\left[r^{2}+\rho^{2}-2 r \rho \cos (\theta-\vartheta)+(z-\zeta)^{2}\right]^{3 / 2}},
\end{aligned}
$$

where $C_{B}=\sigma_{m} /(4 \pi)$. Integrals (7-9) are singular only at the same point as integral (6). 
The model of the magnetic field that leads to equations (7-9) should be able to compute the particular components of $\boldsymbol{B}$ but our goal of preference is $B_{r}$ that forms the main magnetic flux of electric machine. Equation (6) for the scalar magnetic potential is not of the first interest, therefore the computed values of $\psi_{m}$ may be employed for an approximate verifying the computed values of the particular components of $\boldsymbol{B}$ by a convenient finite-difference approach.

\section{Computing the magnetic scalar potential}

Consider firstly equation (6) for $r \neq \rho$, i.e. at field point $\mathrm{P}$ situated outside the sheet on which $\sigma_{\mathrm{m}}$ is homogeneously distributed. The integral can be computed in a mixed way; the first integral with respect to the chosen first variable ( $\zeta$ or $\vartheta$ ) leads to an exact solution, however the numerical integration shall be applied for the second variable.

1) Starting to integrate (6) with respect to variable $\zeta$ (according to Ryshik et al. ${ }^{2}$ ) over the limits $\left(-l_{0},+l_{0}\right)$ we obtain:

$$
\psi_{\mathrm{m}}(r, \theta, z)=C_{\psi} \int_{-\vartheta_{0}}^{\vartheta_{0}} \ln \frac{\sqrt{r^{2}+\rho^{2}-2 r \rho \cos (\theta-\vartheta)+\left(z-l_{0}\right)^{2}-\left(z-l_{0}\right)}}{\sqrt{r^{2}+\rho^{2}-2 r \rho \cos (\theta-\vartheta)+\left(z+l_{0}\right)^{2}-\left(z+l_{0}\right)}} \rho \mathrm{d} \vartheta,
$$

The above integral have not exact solution thus the numerical integration procedure must be applied and the one-dimensional Gaussian quadrature formula is here conformed to.

2) We would like to focus the attention on the second sequence of integration that results in elliptic integrals; that rends to produce the uniform theory of computing as well the magnetic scalar potential as the particular components of the magnetic flux density. If we start to integrate equation (6) with respect to variable $\vartheta$ over limits $\left(-\vartheta_{0},+\vartheta_{0}\right)$ the typical substitution of variable $\varphi=[\pi-(\theta-\vartheta)] / 2$ should be applied that leads to the corresponding integral limits $\varphi_{a}=\left[\pi-\left(\theta+\vartheta_{0}\right)\right] / 2$ and $\varphi_{b}=\left[\pi-\left(\theta-\vartheta_{0}\right)\right] / 2$, and it gives $\cos (\theta-\vartheta)=2 \sin ^{2} \varphi-1$. In consequence, formula (6) can be transformed to the following one:

$$
\psi_{m}=C_{\psi} \int_{-l_{0}}^{l_{0}} \frac{2 \rho}{\sqrt{(r+\rho)^{2}+(z-\zeta)^{2}}} \int_{\varphi_{a}}^{\varphi_{b}} \frac{\mathrm{d} \varphi}{\sqrt{1-k^{2} \sin ^{2} \varphi}} \mathrm{d} \zeta
$$

where

$$
k=2 \sqrt{r \rho} / \sqrt{(r+\rho)^{2}+(z-\zeta)^{2}} .
$$

Interpreting it as a modulus of an elliptic integral the second integral appearing in (11) can be represented by a difference of two incomplete elliptic integrals $\mathrm{F}\left(\varphi_{b}, k\right)$ minus $\mathrm{F}\left(\varphi_{a}, k\right)$, of the first kind of the arguments $\varphi_{a}$ and $\varphi_{b}$, respectively, see Appendix, to read:

${ }^{2}$ See [11] - integral 2.261 . 


$$
\psi_{\mathrm{m}}(r, \theta, z)=C_{\psi} \sqrt{\frac{\rho}{r}} \int_{-l_{0}}^{l_{0}} k\left[\mathrm{~F}\left(\varphi_{\mathrm{b}}, k\right)-\mathrm{F}\left(\varphi_{\mathrm{a}}, k\right)\right] \mathrm{d} \zeta .
$$

If $0 \leq|\theta| \leq \vartheta_{0}$ then $\varphi_{\mathrm{a}} \leq \pi / 2$ but $\varphi_{\mathrm{b}} \leq \pi / 2$ that needs to apply the extension-range formula ${ }^{3}$ to read $\mathrm{F}\left(\varphi_{\mathrm{b}}, k\right)=2 \mathrm{~K}(k)-\mathrm{F}\left(\bar{\varphi}_{\mathrm{b}}, k\right)$, where $\mathrm{K}(k)$ is complete elliptic integral of the first kind of the same modulus and $\bar{\varphi}_{\mathrm{b}}=\pi-\varphi_{\mathrm{b}} \leq \pi / 2$.

If $\vartheta_{0}>|\theta|>\pi / 2$ then both $\varphi_{\mathrm{a}, \mathrm{b}} \leq \pi / 2$ and (12) can be applied, directly.

It is worth to mention that performing a numerical integration of (12) with respect to the variable $\zeta$ the modulus $k$ is taken as depended on the abscissa $u_{i}$ of the Gaussian quadrature to read $k=2 \sqrt{r \rho} / \sqrt{(r+\rho)^{2}+\left(z-u_{i} l_{0}\right)^{2}}$. Thus, the particular $i$-term of quadrature can be evidently computed as function of the elliptic integrals of the first kind of modulus $k_{i}$ and of arguments $\varphi_{\mathrm{a}}$ and $\varphi_{\mathrm{b}}$, or $\bar{\varphi}_{\mathrm{b}}$, respectively, applying the relevant professional software for complete and incomplete elliptic integrals for each Gaussian step.

\section{Computing the magnetic flux density}

Radial component $B_{r}$. Both particular integrals occurring in (7) have analytical solutions and a sequence of them is of any choice. We would like to present the integration with respect to $\zeta$ at first, and then to $\vartheta$. We make use of $[2]^{4}$ to obtain:

$$
B_{r}=\left.C_{B} \int_{-\vartheta_{0}}^{\vartheta_{0}} \frac{[r-\rho \cos (\theta-\vartheta)](z-\zeta) \rho d \vartheta}{\left[r^{2}+\rho^{2}-2 r \rho \cos (\theta-\vartheta)\right] \sqrt{\left[r^{2}+\rho^{2}-2 r \rho \cos (\theta-\vartheta)+(z-\zeta)^{2}\right]}}\right|_{\zeta=-l_{0}} ^{\zeta=l_{0}}
$$

The shortened notice of (13) is the following:

$$
B_{r}=C_{B} \int_{-\vartheta_{0}}^{\vartheta_{0}} \frac{s}{t^{2}}\left[\frac{\left(z-l_{0}\right)}{\sqrt{t^{2}+\left(z-l_{0}\right)^{2}}}-\frac{\left(z+l_{0}\right)}{\sqrt{t^{2}+\left(z+l_{0}\right)^{2}}}\right] \rho \mathrm{d} \vartheta,
$$

where $s=r-\rho \cos (\theta-\vartheta)$ and $t^{2}=r^{2}+\rho^{2}-2 r \rho \cos (\theta-\vartheta)$.

We substitute the variable $\varphi$ in the same way as it is done with respect to the magnetic scalar potential, integrals (11-12), to get the elliptic integrals but in this case both: of the first kind and of the third kind of them are expected. Let us recognize the elliptic integrals, to which the substitution of the new variable leads.

Two similar terms of various sign at $l_{0}$ can be distinguished in (13a). We mark them by the appropriate indices (-) or (+) to read $B_{r}=B_{r(-)}-B_{r(+)}$. Let us discuss a substitution of $\vartheta \rightarrow \varphi$ for $B_{r(-)}$. Consider then

\footnotetext{
${ }^{3}$ See [1] - equation 113.02 .

${ }^{4}$ See [2] - integral 2.264.5.
} 


$$
B_{r(-)}=C_{B} \int_{-\vartheta_{0}}^{\vartheta_{0}} \frac{r-\rho \cos (\theta-\vartheta)}{r^{2}+\rho^{2}-2 r \rho \cos (\theta-\vartheta)} \frac{z-l_{0}}{\sqrt{t^{2}+\left(z-l_{0}\right)^{2}}} \rho \mathrm{d} \vartheta .
$$

After substitution $\varphi=[\pi-(\theta-\vartheta)] / 2$, equation (14) leads to the following formula:

$$
B_{r(-)}\left(\varphi_{1}, \varphi_{2}\right)=\frac{\alpha^{2} k_{(-)}\left(z-l_{0}\right)}{4 r^{3 / 2} \rho^{1 / 2}} \cdot \int_{\varphi_{\mathrm{a}}}^{\varphi_{\mathrm{b}}} \frac{\left(r+\rho-2 \rho \sin ^{2} \varphi\right) \mathrm{d} \varphi}{\left(1-\alpha^{2} \sin ^{2} \varphi\right) \sqrt{1-k_{(-)}^{2} \sin ^{2} \varphi}} .
$$

where:

$$
k_{(-)}^{2}=4 r \rho /\left[(r+\rho)^{2}+\left(z-l_{0}\right)^{2}\right], \quad \alpha^{2}=4 r \rho /(r+\rho)^{2}, \quad \varphi_{\mathrm{a}, \mathrm{b}}=\left[\pi-\left(\theta \pm \vartheta_{0}\right)\right] .
$$

The integrand in (15) consists of a difference of two terms, in fact. The first term determines the difference of two incomplete elliptic integrals of the third kind multiplied by $(r+\rho)$, then

$$
\text { - }(r+\rho)\left[\Pi\left(\varphi_{\mathrm{b}}, \alpha^{2}, k_{(-)}\right)-\Pi\left(\varphi_{\mathrm{a}}, \alpha^{2}, k_{(-)}\right)\right] \text {. }
$$

The second term containing $\sin ^{2} \varphi$ multiplied by $-2 \rho$ leads to, see $[1]^{5)}$

- $\frac{2 \rho}{\alpha^{2}}\left[\Pi\left(\varphi_{\mathrm{a}}, \alpha^{2}, k_{(-)}\right)-\Pi\left(\varphi_{\mathrm{b}}, \alpha^{2}, k_{(-)}\right)+F\left(\varphi_{\mathrm{b}}, k_{(-)}\right)-F\left(\varphi_{\mathrm{a}}, k_{(-)}\right)\right]$.

Before giving the complete formula of $B_{r}=B_{r(-)}-B_{r(+)}$, the following expression on the limits of integral (13a) should be established.

- For $0 \leq|\theta| \leq \vartheta_{0}$ the integral of limits $\left(-\vartheta_{0},+\vartheta_{0}\right)$ can be subdivided into two integrals as follows:

$$
\int_{-\vartheta_{0}}^{\vartheta_{0}} \ldots \mathrm{d} \vartheta=\int_{-\vartheta_{0}}^{\theta-\vartheta_{0}} \ldots \mathrm{d} \vartheta+\int_{\theta-\vartheta_{0}}^{\vartheta_{0}} \ldots \mathrm{d} \vartheta
$$

The integrand of

$$
\int_{\theta-\vartheta_{0}}^{\vartheta_{0}} \ldots \mathrm{d} \vartheta
$$

is a pair function with respect to $\theta$ thus

$$
\int_{\theta-\vartheta_{0}}^{\vartheta_{0}} \ldots \mathrm{d} \vartheta=\int_{\theta-\vartheta_{0}}^{\theta} \ldots \mathrm{d} \vartheta+\int_{\theta}^{\vartheta_{0}} \ldots \mathrm{d} \vartheta=2 \cdot \int_{\theta-\vartheta_{0}}^{\theta} \ldots \mathrm{d} \vartheta
$$

hence, the following sum of integrals with limits expressed by $\varphi_{\mathrm{a}, \mathrm{b}}$ is valid to read:

\footnotetext{
${ }^{5}$ See [1] - integral 337.01 .
} 


$$
\int_{\varphi_{\mathrm{a}}}^{\varphi_{\mathrm{b}}} \ldots \mathrm{d} \varphi=\int_{\varphi_{\mathrm{a}}}^{\varphi_{\mathrm{c}}} \ldots \mathrm{d} \varphi+2 \cdot \int_{\varphi_{\mathrm{c}}}^{\pi / 2} \ldots \mathrm{d} \vartheta,
$$

where $\varphi_{\mathrm{a}}=\left[\pi-\left(\theta-\vartheta_{0}\right)\right] / 2<\pi / 2$, and also $\varphi_{\mathrm{c}}=\left(\pi-\vartheta_{0}\right) / 2<\pi / 2$. Therefore, the above operation leads to confine the elliptic integrals of the third kind to the arguments $\varphi \leq \pi / 2$ that is opportune in view of the computing technique.

- For $\vartheta_{0} \leq|\theta| \leq \pi / 2$ the similar transformation of integral limits is not necessary because both limits are $\varphi_{\mathrm{a}, \mathrm{b}} \leq \pi / 2$.

- Hence we have finally in the case of $0 \leq|\theta| \leq \vartheta_{0}$ :

$$
\begin{aligned}
& B_{r(-)}=C_{B} \frac{k_{(-)}\left(z-l_{0}\right)}{4 r^{3 / 2} \rho^{1 / 2}} \cdot\left\{2 \rho \alpha^{2}\left[2 \mathrm{~K}\left(k_{(-)}\right)-\mathrm{F}\left(\varphi_{\mathrm{a}}, k_{(-)}\right)-\mathrm{F}\left(\varphi_{\mathrm{c}}, k_{(-)}\right)\right]+\right. \\
& \left.+\left[\alpha^{2}(r+\rho)-2 \rho\right]\left[2 \Pi\left(\alpha^{2}, k_{(-)}^{2}\right)-\Pi\left(\varphi_{\mathrm{a}}, \alpha^{2}, k_{(-)}^{2}\right)-\Pi\left(\varphi_{\mathrm{c}}, \alpha^{2}, k_{(-)}^{2}\right)\right]\right\} . \\
& B_{r(+)}=C_{B} \frac{k_{(+)}\left(z+l_{0}\right)}{4 r^{3 / 2} \rho^{1 / 2}} \cdot\left\{2 \rho \alpha^{2}\left[2 \mathrm{~K}\left(k_{(+)}\right)-\mathrm{F}\left(\varphi_{\mathrm{a},} k_{(+)}\right)-\mathrm{F}\left(\varphi_{\mathrm{c}}, k_{(+)}\right)\right]+\right. \\
& \left.+\left[\alpha^{2}(r+\rho)-2 \rho\right]\left[2 \Pi\left(\alpha^{2}, k_{(+)}^{2}\right)-\Pi\left(\varphi_{\mathrm{a}}, \alpha^{2}, k_{(+)}^{2}\right)-\Pi\left(\varphi_{\mathrm{c}}, \alpha^{2}, k_{(-)}^{2}\right)\right]\right\} .
\end{aligned}
$$

- and in the case of $\vartheta_{0} \leq|\theta| \leq \pi / 2$ :

$$
\begin{gathered}
B_{r(-)}=C_{B} \frac{k_{(-)}\left(z-l_{0}\right)}{4 r^{3 / 2} \rho^{1 / 2}} \cdot\left\{2 \rho \alpha^{2}\left[\mathrm{~F}\left(\varphi_{\mathrm{b}}, k_{(-)}\right)-\mathrm{F}\left(\varphi_{\mathrm{a}}, k_{(-)}\right)\right]+\right. \\
\left.+\left[\alpha^{2}(r+\rho)-2 \rho\right]\left[\Pi\left(\varphi_{\mathrm{b}}, \alpha^{2}, k_{(-)}^{2}\right)-\Pi\left(\varphi_{\mathrm{a}}, \alpha^{2}, k_{(-)}^{2}\right)\right]\right\}, \\
B_{r(+)}=C_{B} \frac{k_{(+)}\left(z+l_{0}\right)}{4 r^{3 / 2} \rho^{1 / 2}} \cdot\left\{2 \rho \alpha^{2}\left[\mathrm{~F}\left(\varphi_{\mathrm{b}}, k_{(+)}\right)-\mathrm{F}\left(\varphi_{\mathrm{a}}, k_{(+)}\right)\right]+\right. \\
\left.+\left[\alpha^{2}(r+\rho)-2 \rho\right]\left[\Pi\left(\varphi_{\mathrm{b}}, \alpha^{2}, k_{(+)}^{2}\right)-\Pi\left(\varphi_{\mathrm{a}}, \alpha^{2}, k_{(-)}^{2}\right)\right]\right\} .
\end{gathered}
$$

Thus, the complete and incomplete elliptic integrals of the first and third kind that appear in (17) and (18) are characterized by the squares of moduli are $k_{(-)}^{2}=4 r \rho /\left[(r+\rho)^{2}+\left(z-l_{0}\right)^{2}\right]$, and $k_{(+)}^{2}=4 r \rho /\left[(r+\rho)^{2}+\left(z+l_{0}\right)^{2}\right]$, respectively, the arguments of the incomplete elliptic integrals are $\varphi_{\mathrm{a}}=\left[\pi-\left(\theta+\vartheta_{0}\right)\right] / 2$ and $\varphi_{\mathrm{c}}=\left(\pi-\vartheta_{0}\right) / 2$, the square of parameter of the elliptic integrals of the third kinds is $\alpha^{2}=4 r \rho /(r+\rho)^{2}$. The complete elliptic integrals do not occur in (17a) and (18a), hence, the moduli of incomplete elliptic integrals of the first and third kind are identical like those in (17) and (18), and also identical is the argument $\varphi_{\mathrm{a}}$ but instead of $\varphi_{\mathrm{c}}$ we have here $\varphi_{\mathrm{b}}=\left[\pi-\left(\theta-\vartheta_{0}\right)\right] / 2$.

Azimuthal component $B_{\theta}$. It seems to be reasonable that in the case of the azimuthal component $B_{\theta}$ of $\boldsymbol{B}$ the mixed integration of (8) should be applied: at first, the analytical formula of the integral with respect to variable $\zeta$ should be used, and next - with respect to $\vartheta$ - the numerical integration shall follow it. The first integration leads to the equation: 


$$
B_{\theta}=\left.C_{B} \int_{-\vartheta_{0}}^{\vartheta_{0}} \frac{\rho^{2}(z-\zeta) \sin (\theta-\vartheta) \mathrm{d} \vartheta}{\left[r^{2}+\rho^{2}-2 r \rho \cos (\theta-\vartheta)\right] \sqrt{\left[r^{2}+\rho^{2}-2 r \rho \cos (\theta-\vartheta)-(z-\zeta)^{2}\right]}}\right|_{-l_{0}} ^{l_{0}}
$$

that can be shortly written as:

$$
B_{\theta}=C_{B} \rho^{2} \int_{-\vartheta_{0}}^{\vartheta_{0}} \frac{\sin (\theta-\vartheta)}{t^{2}}\left[\frac{\left(z-l_{0}\right)}{\sqrt{t^{2}+\left(z-l_{0}\right)^{2}}}-\frac{\left(z+l_{0}\right)}{\sqrt{t^{2}+\left(z+l_{0}\right)^{2}}}\right] \rho \mathrm{d} \vartheta .
$$

After transformation of variables $\vartheta \rightarrow \varphi$ as above, we get $\sin (\theta-\vartheta)=\sin (2 \varphi)=$ $=2 \sin \varphi \cos \varphi$ and $t^{2}=(r+\rho)^{2}-4 r \rho \sin ^{2} \varphi$. Hence after simple operations, we obtain the following formula for the both terms of the azimuthal component:

$$
B_{\theta(-)}=C_{B} \cdot \rho^{2} \cdot \int_{\varphi_{1}}^{\varphi_{4}} \frac{2 \sin 2 \varphi}{(r+\rho)^{2}-4 r \rho \sin ^{2} \varphi} \cdot \frac{\left(z-l_{0}\right) \cdot 2 \mathrm{~d} \varphi}{\sqrt{(r+\rho)^{2}-4 r \rho \sin ^{2} \varphi+\left(z-l_{0}\right)^{2}}}
$$

that leads to:

$$
\begin{aligned}
& B_{\theta(-)}=\frac{C_{B} \cdot \rho^{1 / 2} \alpha^{2} k_{(-)}\left(z-l_{0}\right)}{4 r^{3 / 2}} \int_{\varphi_{1}}^{\varphi_{4}} \frac{\sin 2 \varphi \mathrm{d} \varphi}{\left(1-\alpha^{2} \sin ^{2} \varphi\right) \sqrt{1-k_{(-)}^{2} \sin ^{2} \varphi}} \\
& B_{\theta(+)}=\frac{C_{B} \cdot \rho^{1 / 2} \alpha^{2} k_{(+)}\left(z+l_{0}\right)}{4 r^{3 / 2}} \int_{\varphi_{1}}^{\varphi_{4}} \frac{\sin 2 \varphi \mathrm{d} \varphi}{\left(1-\alpha^{2} \sin ^{2} \varphi\right) \sqrt{1-k_{(+)}^{2} \sin ^{2} \varphi}}
\end{aligned}
$$

and then:

$$
B_{\theta}=B_{\theta(-)}-B_{\theta(+)}
$$

The integrals in formulae (21) and (22) have the denominator being typical for elliptical integrals of the third kind. However, the member $\sin 2 \varphi$ appearing in the nominator shatters a chance that an elliptic integral could be here conceivable. Thus, the numerical integration of (23) is a suitable solution to obtain the numerical value of $B_{\theta}$.

Axial component $B_{z}$.In the case of the axial component $B_{z}$ of $\boldsymbol{B}$ for which equation (9) is valid the full analytical solution is possible. The first integration with respect to variable $\zeta$, similarly as in the case of the radial components, leads to the formula ${ }^{6}$ :

$$
B_{z}=C_{B} \cdot \rho\left[\left.\int_{-\vartheta_{0}}^{\vartheta_{0}} \frac{\mathrm{d} \vartheta}{\sqrt{t^{2}+(z-\zeta)^{2}}}\right|_{-l_{0}} ^{l_{0}}+\left.\int_{-\vartheta_{0}}^{\vartheta_{0}} \frac{z(z-\zeta)}{t^{2} \sqrt{t^{2}+(z-\zeta)^{2}}}\right|_{-l_{0}} ^{l_{0}}\right],
$$

\footnotetext{
${ }^{6}$ See [11-integrals 2.264 .5 and 6].
} 
where for $t^{2}=f(\vartheta)$ see a comment to (13a). Substituting the new variables $\vartheta \rightarrow \varphi$, and carrying out necessary operations we obtain the formulae leading to the suitable elliptic integrals to read:

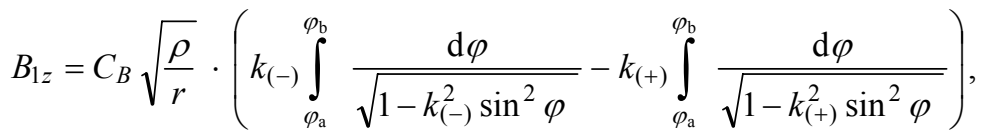

$$
\begin{aligned}
& B_{2 z}=\frac{C_{B} \cdot z \cdot \alpha^{2}}{4 r^{3 / 2} \rho^{1 / 2}} \times \\
& \times\left(\int_{\varphi_{\mathrm{a}}}^{\varphi_{\mathrm{b}}} \frac{k_{(-)}\left(z-l_{0}\right) \mathrm{d} \varphi}{\left(1-\alpha^{2} \sin ^{2} \varphi\right) \sqrt{1-k_{(-)}^{2} \sin ^{2} \varphi}}-\int_{\varphi_{\mathrm{a}}}^{\varphi_{\mathrm{b}}} \frac{k_{(+)}\left(z+l_{0}\right) \mathrm{d} \varphi}{\left(1-\alpha^{2} \sin ^{2} \varphi\right) \sqrt{1-k_{(+)}^{2} \sin ^{2} \varphi}}\right), \\
& B_{z}(r, \theta, z)=B_{1 z}+B_{2 z} .
\end{aligned}
$$

We observe that the elliptic integrals of the first and third kind, to which the integrals occurring in (25) and (26) are to be resolved, are quite similar to those ones being discussed in the case of the radial components of the magnetic flux density. Similarly, the choice of the elliptic integral arguments that are easy to be computed depends in a similar way on the value of $|\theta|$, compare equation (17) and the following ones. The axial component $B_{z}$ disappears at points $\mathrm{P}(r, \theta, 0)$ situated on a surface $(r, \theta)$ being perpendicular and symmetrical to the sheet, because for $z=0$ the term $B_{2 z}$ disappears, evidently. Moreover, for $z=0$ we have equality $k_{(-)}=k_{(+)}$that results in $B_{1 \mathrm{z}}$ becoming zero, too.

\section{Numerical examples}

The test computer programs (coded in LAHEY FORTRAN) were accomplished in order to numerically study the above presented tube segment model provided with $\sigma_{\mathrm{m}}$. The specialized subroutines were programmed for the elliptic integrals. The software published by Press et al [10] has been adjusted to compute the complete elliptic integrals of the first kind $\mathrm{K}(k)$ and second kind $\mathrm{E}(k)$. For the corresponding incomplete elliptic integrals see APPENDIX, where employing the auxiliary Heuman's lambda function for computing the elliptic integrals of the third kind has also been described.

The curves shown in Figures 4, 5 and 6 are related to the single test sheet upon which the $\sigma_{\mathrm{m}}=1 \mathrm{~T}$ is homogeneously distributed. The sheet is characterized by radius $\rho=100 \mathrm{~mm}$, extension arc $2 \vartheta_{0}=80^{\circ}$ and length $2 l_{0}=80 \mathrm{~mm}$.

The radial distribution of the magnetic scalar potential along the line passing through the sheet center and perpendicular to it is given in Figure 4. The values of $\psi_{\mathrm{m}}$ have been chosen at a distance of $-10 \mathrm{~mm}$ to $+10 \mathrm{~mm}$ from the sheet surface. We observe that the values at the concave side of the sheet surpass those on the convex side. This result seems to be obvious in the light of the fact that the resulting distance from the totality of the source points $\mathrm{Q}$ to the 
field points $\mathrm{P}$ is shorter for $\mathrm{P}$ being situated at the concave side of the sheet than for $\mathrm{P}$ at the convex side. For $\mathrm{P}(\rho, 0,0)$ situated exactly at the center of the sheet both curves meet together, indeed. This common value of the magnetic scalar potential cannot be computed directly by the formulae either (10) or (12) because of singularity. We have obtained the corresponding values after performing a two-side extrapolation on the basis of the computed values at the vicinity to $\mathrm{P}(\rho, 0,0)$ from both sides of the sheet.

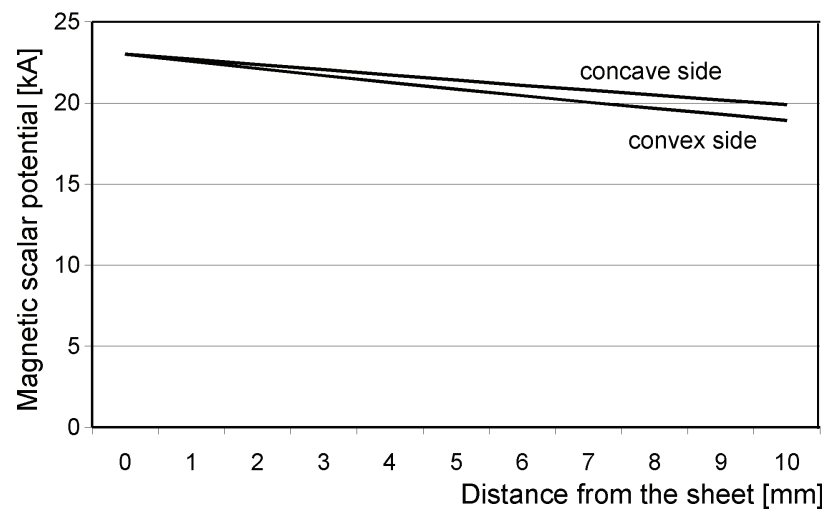

Fig. 4. Functions $\psi_{m}=f(|r-\rho|)$ of the test sheet of data: $\rho=100 \mathrm{~mm}, 2 \vartheta_{0}=80^{\circ}, 2 l_{0}=80 \mathrm{~mm}$ field points $|r-\rho| \in(0 ; 10) \mathrm{mm}, \theta=0^{\circ}, z=0 \mathrm{~mm}$

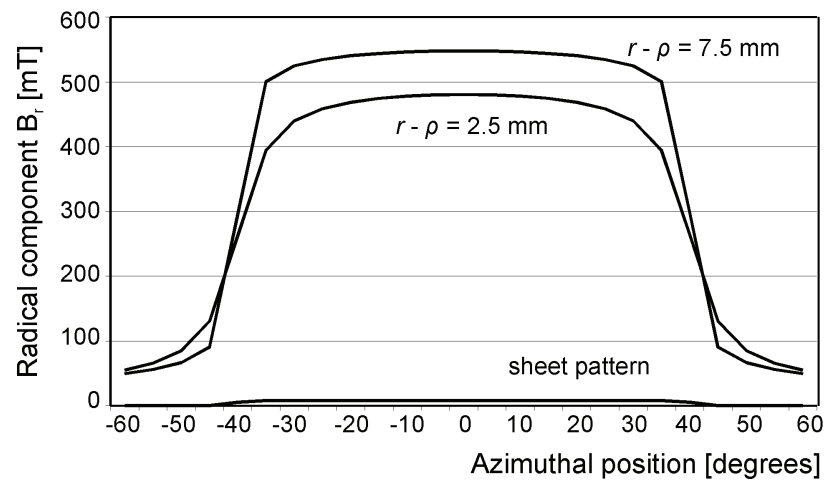

Fig. 5. Functions $B_{r}=f(\theta)$; for the same test sheet as in Figure 4; field points $|r-\rho|=2,5 \mathrm{~mm}$ and $7,5 \mathrm{~mm} \theta \in\left(-60^{\circ},+60^{\circ}\right), z=0 \mathrm{~mm}$

The azimuthal distribution of the radial component of the magnetic flux density (at the convex side of the same sheet) is computed following formulae (17), (18) or in the occurrence of (17a), (18a) is presented in Figure 5. Over the sheet surface i.e. for $\theta \in\left(-40^{\circ},+40^{\circ}\right)$ the pattern of which is marked, the curves decline slightly from their maximal values at $\mathrm{P}(\rho+2,5,0,0)$ or $\mathrm{P}(\rho+7,5,0,0)$, respectively, but they are strongly declined at $\theta$ taking values outside the above interval. 
A coincidence of the curve $B_{r}=f(|\rho-r|)$ with curve $\psi_{\mathrm{m}}=f(|r-\rho|)$ may be verified at some field points of the curve, the values of which were computed similarly as those being raced in Figure 5, e.g. at point $\mathrm{P}_{0}(102,5,0,0)$ to what $B_{r}=547,9 \mathrm{mT}$ corresponds. The values of $\psi_{\mathrm{m}}$ at two points near to $\mathrm{P}_{0}$ are $\psi_{\mathrm{m} 1}=f(103 ; 0 ; 0)=21,964 \mathrm{kA}$ and $\psi_{\mathrm{m} 2}=f(102 ; 0 ; 0)=$ $=22,995 \mathrm{kA}$, see Figure 4. If we apply the central finite difference formula for $\Delta r=103,0-$ $-102,0=1 \mathrm{~mm}$ we obtain approximately $B_{r} \approx \mu_{0} \cdot 10^{6} \cdot(22.995-21.964) / 1=547.9 \mathrm{mT}$. The coincidence of the numerical value of $B_{r}$ (computed directly by formulae using the elliptic integrals) with its approximate value obtained indirectly by way of numerical values of $\psi_{\mathrm{m}}$ seems to argue for practical applying the formulae based on the elliptic integrals that we evaluate to be characteristic for the mathematical model of the magnetic field exerted by the permanent magnet of tube segment as shape.

Figure 6 shows the azimuthal distribution of the azimuthal component $B_{\theta}=f(\theta)$ for the convex side of the same sheet. The curves are traced for the two various values of $r-\rho$ and for $z=0$. The curve is asymmetrical with respect to the line $\theta=0$. The maximum values correspond to the sheet edges.

Figure 7 shows the computed values of the norm $|\boldsymbol{B}|$ (continuous line) at the surface $(r, z)$ and its two components $B_{r}$ and $B_{z}$ (dashed lines) as functions of the longitudinal coordinate $z$ at $\theta=0$, and $r-\rho=5,0 \mathrm{~mm}$. The third co-ordinate $B_{\theta}$ disappears at this surface. The norm of the magnetic flux density is not evidently influenced by its longitudinal component at most part of the region over the magnet. This is not the case at the magnet edge regions and also outside the magnet region.

The following examples presented in Figures 8 and 9 are related to the permanent magnet of the tube segment as shape of the data: $\rho_{\mathrm{N}}=130 \mathrm{~mm}, h=6.5 \mathrm{~mm}$, then $\rho_{\mathrm{S}}=123.5 \mathrm{~mm}$, $2 \vartheta_{0}=12^{\circ}, 2 l_{0}=85 \mathrm{~mm}$. The magnet was installed into the multi-pole synchronous machine that was designed and manufactured in the Electrotechnical Institute in Warsaw. The typical magnetization data are $B_{\mathrm{rem}}=1,23 \mathrm{~T}, H_{\mathrm{co}}=965 \mathrm{kA} / \mathrm{m}$.

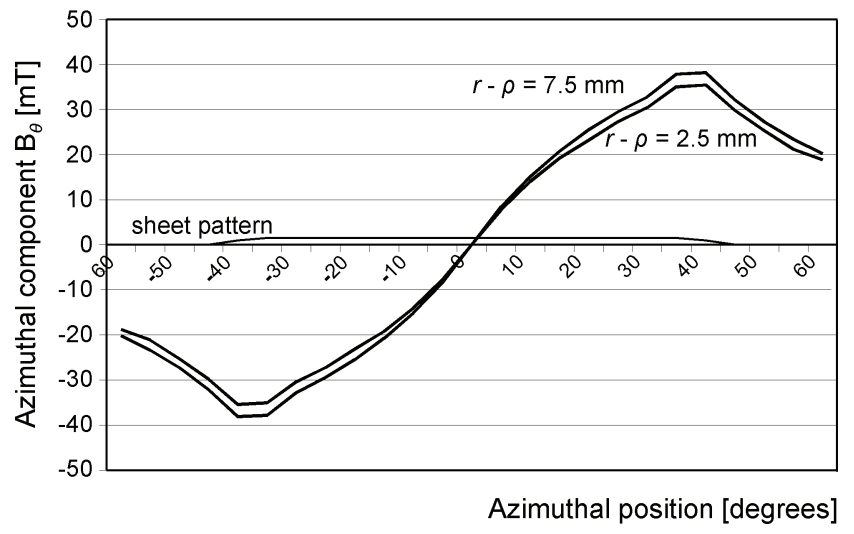

Fig. 6. Function $B_{\theta}=f(\theta)$ exerted at a convex side of the single sheet of data: $\rho=100 \mathrm{~mm}, 2 \vartheta_{0}=80^{\circ}$, $2 l_{0}=80 \mathrm{~mm}, \sigma_{\mathrm{m}}=1.0 \mathrm{~T}$; coordinates of the field points $r-\rho=2.5 \mathrm{~mm}$ and $7.5 \mathrm{~mm}, \theta \in\left(-60^{\circ},+60^{\circ}\right)$, $z=0.0 \mathrm{~mm}$. 


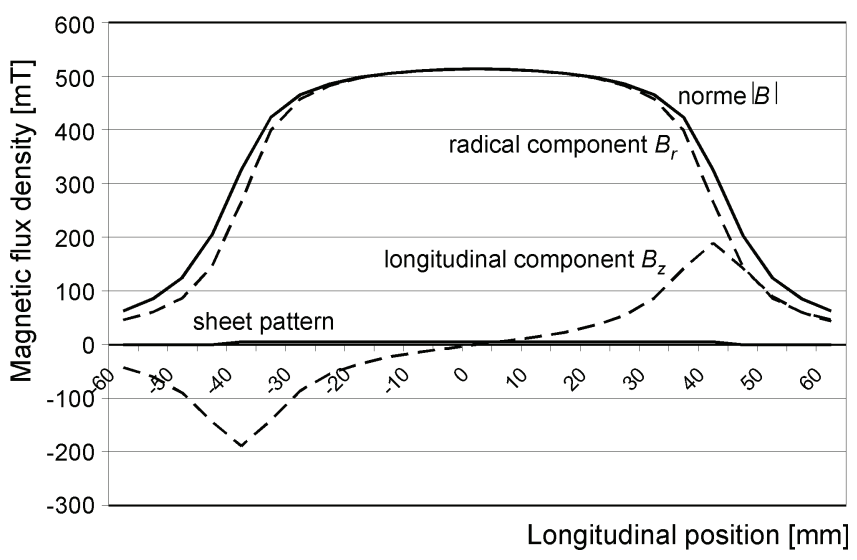

Fig. 7. Function $|\boldsymbol{B}|=f(z)$ and their components $B_{z}=f(z)$ and $B_{r}=f(z)$ exerted at a convex side of the single sheet of data: $\rho=100 \mathrm{~mm}, 2 \vartheta_{0}=80^{\circ}, 2 l_{0}=80 \mathrm{~mm}, \sigma_{\mathrm{m}}=1,0 \mathrm{~T}$; the coordinates of the field points are $r-\rho=5.0 \mathrm{~mm}, \theta=0.0^{\circ}, \mathrm{z} \in(-60 \mathrm{~mm},+60 \mathrm{~mm})$

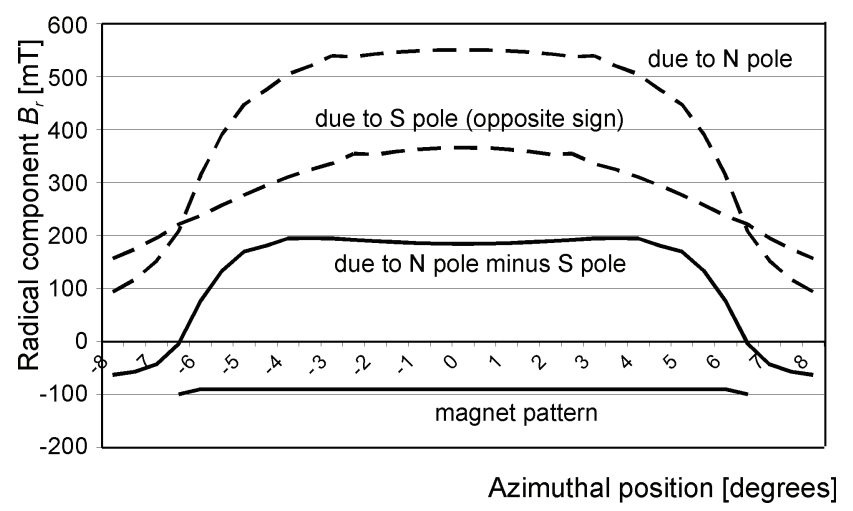

Fig. 8. Function $B_{r}=f(\theta)$ exerted by permanent magnet at its convex side, magnet data: $\rho_{\mathrm{N}}=130 \mathrm{~mm}, \rho_{\mathrm{S}}=123.5 \mathrm{~mm}, 2 \vartheta_{0}=12^{\circ}, 2 l_{0}=85 \mathrm{~mm} ; B_{\mathrm{rem}}=1.23 \mathrm{~T}, H_{\mathrm{co}}=965 \mathrm{kA} / \mathrm{m}$; field points: $r-\rho_{\mathrm{N}}=2.5 \mathrm{~mm}, \theta \in\left(-8^{\circ},+8^{\circ}\right), z=0 \mathrm{~mm}$

The pattern of the magnet spread out from $-42,5 \mathrm{~mm}$ to $+42.5 \mathrm{~mm}$ is marked. The curves presented are computed under supposition that the magnet is put in free space, hence its both poles give contributions to the total results. The figures present the azimuthal distributions of the radial component $B_{r}=f(\theta)$ due to the above permanent magnet at its convex and concave sides: Figures 8 and 9, respectively. The dashed curves correspond to the particular N-pole or S-pole contributions of the magnet and the continoues curves represent the resulting radial component of the magnetic flux density $B_{r}=B_{r \mathrm{~N}}-B_{r \mathrm{~s}}$. Observe, that maximum values of the resulting distributions are localized not at $\theta=0$ but, approximately, over the edges of the magnet. It is due to the fact that the closed $B$-lines passing through the magnet centre are characterised by the longer path than the lines passing near the magnet edge surface. To the longer path the increased value of the magnetic potential difference corresponds that results in some decreasing of the magnetic flux density at the magnet centre. The fact that the computed 
values reflect such a correctness of the distribution $B_{r}=f(\theta)$ testifies in favour of the presented here theory based on the elliptic integrals that expresses the magnetic field engendered by the permanent magnet of a tube segment as shape.

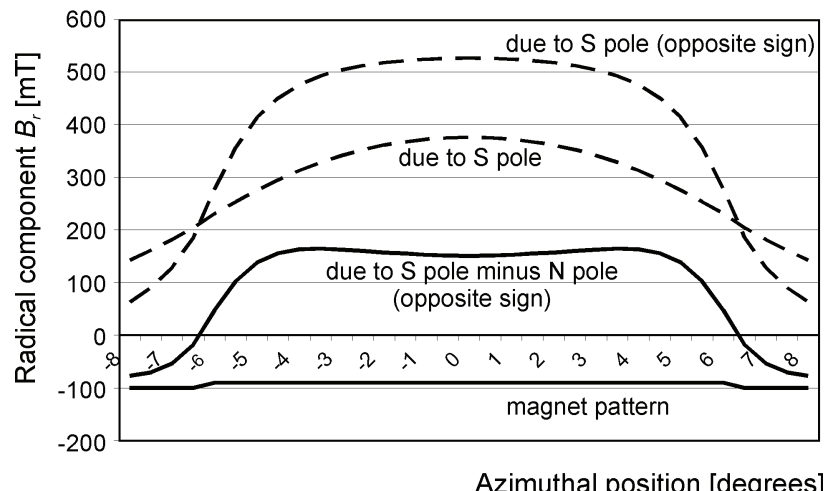

Fig. 9. Function $B_{r}=f(\theta)$ exerted by the permanent magnet at its concave side, magnet data see Fig. 7; field points: $\rho_{\mathrm{S}}-r=2.5 \mathrm{~mm}, \theta \in\left(-8^{\circ},+8^{\circ}\right), z=0 \mathrm{~mm}$

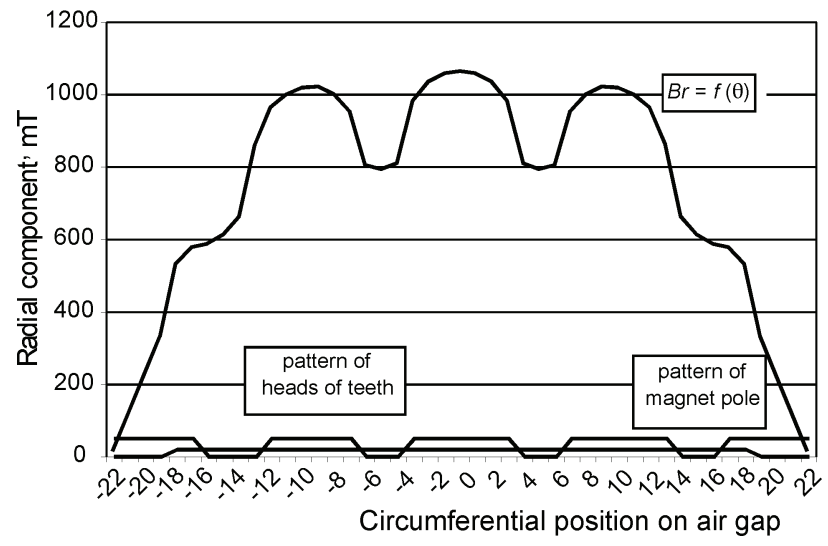

Fig. 10. Function $B_{r}=f(\theta)$ within the air gap of a permanent-magnet synchronous generator at no-load; 1 point of the circumferential position corresponds to $1 / 3 \mathrm{deg}$

The last numerical example concerns the generator with permanent magnets designed and made in the Electrotechnical Institute in Warsaw. The main data of the generator are:

- $P=1 \mathrm{~kW} ; U=52 \mathrm{~V} ; I=11,7 \mathrm{~A} ; f=20 \mathrm{~Hz} ; n=100 \min ^{-1} ; 2 p=12 ; Q_{\mathrm{s}}=108$.

Main sizes:

- Rotor diameter $D_{\mathrm{r}}=130 \mathrm{~mm}$; air-gap thickness $\delta=1.0 \mathrm{~mm}$, pole pitch $\tau_{p}=15^{\circ}$; slot pitch $\tau_{Q} 3,33^{\circ}$; head of teeth $=2,33^{\circ}$.

The permanent magnet of data ${ }^{7} B_{\mathrm{rem}}=1.23 \mathrm{~T}, H_{\mathrm{co}}=965 \mathrm{kA} / \mathrm{m}, h=6.5 \mathrm{~mm}, 2 \vartheta_{0}=12^{\circ}$ are glued to the yoke surface.

${ }^{7}$ The same magnet data like for the two precedent examples. 
- Rapport of the pole pitch to the slot pitch was chosen to $9 / 2$ i.e. being equal to a fraction number with intention to prevent a cogging torque of the generator.

Figure 10 shows the computed distribution of the radial component of magnetic flux density within the air gap at a level of $0.5 \delta$ for the symmetric position of the tooth axis relatively to magnet pole. The test computer programs that have been used to compute the data for the two last examples have been ad hoc adapted for the case of the magnetic field within the air gap of the generator. The magnetic field was computed at 45 points of a half of pole pitch. The region of teeth and slots are marked in the figure as well as a pattern of the magnet pole.

\section{Final remarks}

The main idea leading to determine the magnetic field produced by a permanent magnet of a tube segment as shape was inspired by the need to study the magnetic flux density within an air gap of electric machines equipped with such magnets. The main formulae expressing the scalar magnetic potential and three cylindrical components of magnetic flux density were formulated by the double integral over a pole surface of the magnet. Analytical solutions of those integrals lead to resulting formulae with the elliptic integrals. Numerical examples have shown that such an approach can be applied for computing a distribution of the magnetic flux density within an air gap of electric machines with permanent magnets. The corresponding software appropriate to perform the relevant computing can be coded on the basis of the mathematic formulae presented in the paper. This relatively simply software may be useful for designers of the electric machines in completing project calculations with the distribution of the particular components of magnetic flux density within the air gap. Such a specialized program may be applied instead of the professional field-distribution software that are largely supplied on the computer markets but the use of which needs to do a lot of pre-processing works and to overcome difficulties resulting from the fact that majority of the professional field software operate with various physical fields and are not specialized for the magnetic fields.

\section{Acknowledgements}

The authors would like to thank dr. eng. Zdzisław Krzemien with the Department of Electric Machines in the Electrotechnical Institute, Warsaw, for agreement to publish the data of the generator, which he has designed and tested.

\section{Appendix - guide to elliptic integrals}

The symbols of elliptic integrals in normal trigonometric notation conform to Byrd \& Friedman monograph [1] are applied in the paper and listed below:

- $\varphi, \varphi_{\mathrm{a}}, \varphi_{\mathrm{b}}, \varphi_{\mathrm{c}}$ - arguments (general and particular) of the elliptic integrals; that are generally defined for $\varphi$, placing values at the closed interval $[0 ; \pi / 2]$,

- $k$ - modulus of the elliptic integrals, generally $0<k<1$,

- $k^{\prime}=\sqrt{1-k^{2}}-$ complementary modulus of the elliptic integrals, 
- $\alpha^{2}$-square of a characteristic parameter of the elliptic integral of the third kind,

- $\mathrm{F}(\varphi, k)=\int_{0}^{\varphi} \frac{\mathrm{d} \varphi}{\sqrt{1-k^{2} \sin ^{2} \varphi}}-$ incomplete elliptic integral of the first kind,

- $\mathrm{F}(\pi / 2, k) \equiv \mathrm{K}(k)-$ is said to be the complete elliptic integral of the first kind,

- $\mathrm{E}(\varphi, k)=\int_{0}^{\varphi} \sqrt{1-k^{2} \sin ^{2} \varphi} \mathrm{d} \varphi-$ incomplete elliptic integral of the second kind,

- $\mathrm{E}(\pi / 2, k) \equiv \mathrm{E}(k)$ - is said to be the complete elliptic integral of the second kind,

- $\Pi\left(\varphi, \alpha^{2}, k\right)=\int_{0}^{\varphi} \frac{\mathrm{d} \varphi}{\left(1-\alpha^{2} \sin ^{2} \varphi\right) \sqrt{1-k^{2} \sin ^{2} \varphi}}-$ incomplete elliptic integral of the thir kind,

- $\Pi\left(\pi / 2, \alpha^{2}, k\right) \equiv \Pi\left(\alpha^{2}, k\right)$ - is said to be the complete elliptic integral of the third kind.

The incomplete elliptic integrals of both the first and the second kind can be also expressed for the argument $\varphi$ placing values outside the interval $[0, \pi / 2]$. For instance:

- $\mathrm{K}(-\varphi, k)=-\mathrm{K}(\varphi, k)$, and a similar formula $\mathrm{E}(-\varphi, k)=-\mathrm{E}(\varphi, k)$,

- $\mathrm{K}(\pi \pm \varphi, k)=2 \mathrm{~K}(k) \pm \mathrm{K}(\varphi, k)$, and similarly $\mathrm{E}(\pi \pm \varphi, k)=2 \mathrm{E}(k) \pm \mathrm{E}(\varphi, k)$.

A general formula expressing the incomplete elliptic integral of the third kind for the argument $\varphi$ taking values outside their basic interval does not exist.

The elliptic integrals of both the first and second kind are largely tabulated. This form is, however, not useful for a computer design. Various professional software for the elliptic integrals are accessible on software markets. All of them are based upon some infinite series, e.g. the complete elliptic integral of the first kind for $k^{2}<1$ and $0<\varphi<\pi / 2$ can be computed by the hypergeometric series $\mathrm{F}(\alpha, \beta ; \gamma ; z)$ to get:

- $\mathrm{K}(k)=\frac{\pi}{2} \mathrm{~F}\left(1 / 2,1 / 2 ; 1 ; k^{2}\right)$,

- $\mathrm{E}(k)=\frac{\pi}{2} \mathrm{~F}\left(-1 / 2,1 / 2 ; 1 ; k^{2}\right)$.

For the numerical example given in the paper the software published by Press et al [10] has been adjusted to compute the complete elliptic integrals $\mathrm{K}(k)$ and $\mathrm{E}(k)$. For the incomplete elliptic integrals of the first and the second kind we used the following series given by Byrd and Friedman [2]:

- $\mathrm{F}(\varphi, k)=\sum_{m=0}^{\infty}\left(\begin{array}{c}-1 / 2 \\ m\end{array}\right)\left(-k^{2}\right) t_{2 m}(\varphi)$,

- $\mathrm{E}(\varphi, k)=\sum_{m=0}^{\infty}\left(\begin{array}{c}1 / 2 \\ m\end{array}\right)\left(-k^{2}\right) t_{2 m}(\varphi)$,

where:

- $t_{0}=\varphi ; \quad t_{2}=\frac{1}{2}(\varphi-\sin \varphi \cos \varphi) ; \quad t_{4}=\frac{1}{8}\left[3 \varphi-\left(3+2 \sin ^{2} \varphi\right) \sin \varphi \cos \right]$,

- $t_{2 m}=\frac{2 m-1}{2 m} t_{2(m-1)}-\frac{1}{2 m} \sin ^{2 m-1} \varphi \cos \varphi$. 
It is essential that in order to compute the magnetic scalar potential and the components of the magnetic flux density at the vicinity of the magnetizing sheet (e.g. within an air gape of electric machines) the software shall be clear-sightedly chosen. It should be necessary to compute the elliptic integrals correctly even for $k^{2}$ approaching closely to 1 . Our experience, which we have gained using the series given above, showed that they converge rather slowly and a high number of several hundred series terms should be included into reckoning.

In order to compute values of the elliptic integral of the third kind, we prefer to use two intermediate functions $\Lambda_{0}$ and $\Omega_{0}$. The first of them is termed Heuman's lambda function being defined as follows:

$$
\text { - } \Lambda_{0}(\beta, k)=\frac{2}{\pi}\left[\mathrm{E}(k) \mathrm{F}\left(\beta, k^{\prime}\right)+\mathrm{K}(k) \mathrm{E}\left(\beta, k^{\prime}\right)-\mathrm{K}(k) \mathrm{F}\left(\beta, k^{\prime}\right)\right] \text {, }
$$

where $\mathrm{F}\left(\beta, k^{\prime}\right)$ and $\mathrm{E}\left(\beta, k^{\prime}\right)$ are associated elliptic integrals of the first and second kind, respectively. To $\Omega_{0}$ an own term is not attributed.

The relevant procedure leading to the values of the complete elliptic integrals of the third kind is the following one:

- The first step consist in determining the value of the argument $\beta$ that depends on the parameters $k, k^{\prime}, \alpha^{2}$ and on a mutual relation between them. For the values of the above parameters that we deal according to the model shown in Figure 3, the typical relation $k^{2}<\alpha^{2}<1$ is valid, for which

$$
\beta=\sin ^{-1} \frac{\sqrt{\alpha^{2}-k^{2}}}{\alpha k^{\prime}} .
$$

- In the second step, the Heuman's function $\Lambda_{0}(\beta, k)$ has to be computed conform to its basic definition given above that makes use on the relevant values of the elliptic integrals of the first and second kinds.

- The third step, the final equation of the complete elliptic integral of the third kind is computed by the equation:

$$
\Pi\left(\alpha^{2}, k\right)=\frac{\alpha \pi \Lambda_{0}(\beta, k)}{2 \sqrt{\left(\alpha^{2}-k^{2}\right)\left(1-\alpha^{2}\right)}} .
$$

The incomplete elliptic integrals can be calculated in accordance with the same general idea but we need to distinguish two detailed cases: I: $\left(k^{2}<\alpha^{2}<k<1\right)$ and II: $\left(k^{2}<k<\alpha^{2}<1\right)$ In the case I the argument $\beta$ is identical as given above for the complete elliptic integral of the third kind, and in the case II it is equal to $\beta=\sin ^{-1} \sqrt{1-\alpha^{2}} / k^{\prime}$. The equations of the incomplete elliptic integral are:

- case I: $\Pi\left(\varphi, \alpha^{2}, k\right)=\frac{\alpha \pi\left[\mathrm{F}(\varphi, k) \Lambda_{0}(\beta, k)-\Omega_{\mathrm{I}}\right]}{2 \mathrm{~K}(k) \sqrt{\left(\alpha^{2}-k^{2}\right)\left(1-\alpha^{2}\right)}}$,

- case II: $\Pi\left(\varphi, \alpha^{2}, k\right)=\mathrm{F}(\varphi, k)+\frac{\alpha \pi\left[\Omega_{\mathrm{II}}-\mathrm{F}(\varphi, k) \Lambda_{0}(\beta, k)\right]}{2 \mathrm{~K}(k) \sqrt{\left(\alpha^{2}-k^{2}\right)\left(1-\alpha^{2}\right)}}$, 
in which the formulae $\Omega_{\mathrm{I}}$ and $\Omega_{\mathrm{II}}$ can be calculated by the following infinite series, that converge very fast, generally:

$$
\begin{aligned}
& \text { - case I: } \Omega_{\mathrm{I}}=\frac{2 \mathrm{~K}(k)}{\pi} \tan ^{-1}\left[\frac{2 \sum_{m=1}^{\infty} q^{m^{2}} \sin (2 m v) \sinh (2 m w)}{1+2 \sum_{m=1}^{\infty} q^{m^{2}} \cos (2 m v) \cosh (2 m w)}\right], \\
& \text { - case II: } \Omega_{\mathrm{II}}=F(\varphi, k)-\frac{2 \mathrm{~K}(k)}{\pi} \tan ^{-1}\left[\frac{2 \sum_{m=1}^{\infty} q^{m^{2}} \sin (2 m v) \sinh [2 m(p-w)]}{1+2 \sum_{m=1}^{\infty} q^{m^{2}} \cos (2 m v) \cosh [2 m(p-w)]}\right] \text {, }
\end{aligned}
$$

where: $v=(\pi \mathrm{F}(\varphi, k)) /(2 \mathrm{~K}(k)) ; w=\left(\pi \mathrm{F}\left(\beta, k^{\prime}\right)\right) /(2 \mathrm{~K}(k)) ; p=\left(\pi \mathrm{K}\left(k^{\prime}\right)\right) /(2 \mathrm{~K}(k)) ; q=\mathrm{e}^{-2 p}$.

We have performed a number of numerical experiments to compare the values of the elliptic integrals of the third kind of both complete and incomplete type. We have examined the problem of numerical accuracy of the results obtained by various approaches. In particular, the results reached from the software published in [10] have been compared with the results obtained following the procedure suggested above, and, more over, some special values of $\Pi\left(\varphi, \alpha^{2}, k\right)$ for $\alpha^{2}=k^{2}$ having exact solution were considered.

The main conclusion related to the procedure leading to the most exact computed results of the elliptic integrals of the third kind occurring within the framework of the theory of the magnetic field produced by the permanent magnet of a tube segment as shape is that one, which we presented above. The case where both $k^{2}$ and $\alpha^{2}$ are approaching to 1 seems to be the critical point for computing the correct values of $\Pi\left(\alpha^{2}, k\right)$ and $\Pi\left(\varphi, \alpha^{2}, k\right)$. The computer programs performing the relevant calculations should be coded on the basis of numbers expressed by minimum 16 decimal digits, e.g. in the technique of double precision in FORTRAN-LAHEY.

\section{References}

[1] Byrd P.F., Friedman M.D., Handbook of elliptic integrals for engineers and students. Springer-Verlag, Berlin (1971).

[2] Craik D., Magnetism, principles and applications. J. Viley \& Sons, New York.

[3] Pawluk K., Scalar boundary-integral model of the 3-dimentional magnetic field. Proceedings of Electrotechnical Institute 158: 5-41 (1990) (in Polish).

[4] Pawluk K., 3-D magnetic field of coils with an open magnetic core in boundary-integral approach, in Boundary element technology VIII. Comp. Mech. Publ., Southampton, Boston, pp. 147-156 (1993).

[5] Pawluk K., Życki Z., Permanent magnet within a ferromagnetic structure - boundary-integral model and its experimental verification. Archives of Electrical Engineering 3-4: 273-288 (2006).

[6] Pawluk K., Algorithms based on integrals of Lipschitz-Hankel type for modelling permanent magnet fields. Bull. of the Polish Academy of Sciences, Technical Sciences 49/4, pp. 567-579 (2001).

[7] Pawluk K., Integrals of Lipschitz-Hankel type in analysis of magneto-static fields. Journal of Technical Physics 44(2): 133-144 (2003).

[8] Pawluk K., Permanent magnet field calculated by the boundary-integral approach. Archives of Electrical Engineering 3-4: 257-276 (2008). 
[9] Pawluk K., Exact solutions of integrals to be used in computing the magnetic field by boundaryintegral technique. Proceedings of Electrotechnical Institute 241: 9-27 (2009).

[10] Press W., Flannery B., Teukolsky S., The art of scientific computing. Cambridge University Press, Cambridge (1968).

[11] Ryshik I.M., Gradstein I.S., Tables of series, products and integrals. VEB Deutscher Verlag der Wissenschaften, Berlin (1957).

[12] Tozoni O.W., Method of secondary sources in electric technology. Energia, Moskow (1975) (in Russian) 